\title{
Vogt-Koyanagi-Harada disease
}

INSERM

\section{Source}

INSERM. (1999). Orphanet: an online rare disease and orphan drug data base. VogtKoyanagi-Harada disease. ORPHA:3437

Vogt-Koyanagi-Harada disease is a bilateral, chronic, diffuse granulomatous panuveitis typically characterized by serous retinal detachment and frequently associated with neurological (mening itis), auditory, and dermatological alterations. 